\title{
The cataclysmic variable CW $1045+525$ : A secondary-dominated dwarf nova?
}

\author{
C. Tappert ${ }^{1,2}$, J. R. Thorstensen ${ }^{3}$, W. H. Fenton ${ }^{3}$, N. Bennert ${ }^{4}$, L. Schmidtobreick ${ }^{5,6}$, and A. Bianchini ${ }^{2}$ \\ 1 Departamento de Física y Matemáticas, Grupo de Astronomía, Universidad de Concepción, Casilla 160-C, \\ Concepción, Chile \\ 2 Dipartimento di Astronomia, Università di Padova, Vicolo dell'Osservatorio 2, 35122 Padova, Italy \\ 3 Department of Physics and Astronomy, Dartmouth College, Hanover, New Hampshire, 03755 USA \\ 4 Astronomisches Institut, Ruhr-Universität, 44780 Bochum, Germany \\ 5 European Southern Observatory, Casilla 19001, Santiago 19, Chile \\ 6 Osservatorio Astronómico di Padova, Vicolo dell'Osservatorio 5, 35122 Padova, Italy
}

Received 12 June 2001 / Accepted 10 October 2001

\begin{abstract}
We present spectroscopic and photometric observations of the cataclysmic variable CW 1045+525. Both the optical spectrum and the photometric lightcurve show a strong contribution of a K5V-M0V secondary. We derive an orbital period $P_{\text {orb }}=0.271278(1)$ d by measuring the radial velocities of the absorption lines of the secondary. The period and spectral type of the secondary suggest a distance of 350-700 pc. There is evidence for additional sources of line- and continuum emission, but no direct evidence of an accretion disc. We discuss several scenarios for the nature of CW $1045+525$ on the basis of our results, finding a dwarf nova classification to be the most probable, although not completely satisfying, explanation for the observed characteristics.
\end{abstract}

Key words. stars: individual: CW 1045+525 - stars: novae, cataclysmic variables - stars: fundamental parameters binaries: general

\section{Introduction}

Cataclysmic variables (CVs) are close binaries consisting of a white dwarf primary and usually a late-type mainsequence secondary. The latter fills its Roche lobe and thus enables mass transfer into the gravitational regime of the primary. Due to the conservation of angular momentum, this transfer - in the absence of strong magnetic fields - takes place via an accretion disc. For a comprehensive overview on CVs see Warner (1995).

The little-studied object CW $1045+525$ was discovered as part of the Case Low-Dispersion Northern Sky Survey. It showed strong Balmer emission lines superimposed on a late-type spectrum, but with a very strong ultraviolet continuum. Pesch \& Sanduleak (1987) drew attention to the object, noting that the spectrum was quite unusual. Wagner et al. (1988) and Szkody \& Howell (1992) both published spectra which show the broad Balmer and HeI emission characteristic of cataclysmic binaries, together with very strong K-star features. The object is listed as "UMa5" in the Downes \& Shara (1993) atlas. Henden \& Honeycutt (1995) published a photometric sequence for the field. Although the system is generally

Send offprint requests to: C. Tappert, e-mail: claus@gemini.cfm.udec.cl classified as a "dwarf nova", we were unable to find any indication that outbursts have been observed.

We here present photometric time series and timeresolved spectroscopy of CW $1045+525$. Preliminary evaluations of part of this data have been presented by Bennert et al. (1999) and Tappert et al. (2000).

\section{Observations and reduction}

The 1998 and 1999 data were obtained during the Astronomisches Beobachtungspraktikum (e.g., Schmidtobreick et al. 2000) at the $1 \mathrm{~m}$ Cassegrain reflector of the Hoher List Observatory $^{1}$, Germany. The telescope was equipped with a Ford Loral FA2048 CCD and a Johnson $V$ filter. The CCD is read out through two different channels, thus minimizing read-out time, but making a separate bias correction for both halfs of the CCD necessary. Apart from that, the reduction was done in the standard way, using $\mathrm{IRAF}^{2}$ tasks to apply overscan and domeflats.

The 2000 January observations were taken by JRT using the 2.4-m Hiltner telescope at MDM Observatory, Kitt Peak, Arizona, USA, with the modular spectrograph,

\footnotetext{
1 The Hoher List Observatory is operated by the University of Bonn.

${ }^{2}$ IRAF is distributed by the National Optical Astronomy Observatories.
} 
and the same equipment and protocols were used by WHF for the 2001 March and 2001 May observations. For the MDM observations we observed comparison lamps frequently, and checks of the $\lambda 5577$ night-sky line show that our wavelength scale is typically stable to $\sim 5 \mathrm{~km} \mathrm{~s}^{-1}$. When the weather appeared photometric we observed flux standards in twilight. Even so, our absolute fluxes are not expected to be accurate to much better than 30 per cent, because of occasional clouds and variable losses at the spectrograph slits ( $1^{\prime \prime}$ at the $2.4 \mathrm{~m}$ ). Furthermore, for unknown reasons the modular spectrograph produces wavelike distortions in the continua; these appear to average out in sums of many exposures.

The MDM data were reduced to counts vs. wavelength at the observatory, using standard IRAF procedures. Radial velocities of emission lines were measured using convolution algorithms described by Schneider \& Young (1980) and Shafter (1983). For absorption velocities, we used the cross-correlation algorithms of Tonry \& Davis (1979), as implemented in the rvsao package (Kurtz \& Mink 1998). To search for periods we used the "residualgram" method described by Thorstensen et al. (1996), and to test alias choices in doubtful cases we used the Monte Carlo method explained by Thorstensen \& Freed (1985).

In March and April 2000 the system was observed with the $1.82 \mathrm{~m}$ telescope at Mt. Ekar, Asiago Observatory, Italy. The AFOSC $^{3}$ system was used, thus enabling both spectroscopic and photometric measurements without changing the instrumental configuration. In March we used a red grism (\#8) for time-resolved spectroscopy and $B, V, R$ filters for accompanying time-series photometry. The sequence of the measurements was chosen to be spectrum $-B-V-R$, in an attempt to compare radial velocities with the lightcurve, and to gather information on the different components which contribute to the latter. However, poor weather conditions causing long integration times and frequent gaps spoiled that effort. For the April observations we therefore chose to concentrate on the spectroscopy and opted for a sequence of four spectroscopic measurements followed by one image in the $V$ passband. The spectroscopy was done by alternating between a blue $(\# 7)$ and a red grism (\#8). Every measurement was followed by corresponding wavelength calibration exposures (a He lamp for grism \#7, Ne for grism \#8). As in March, the weather was not photometric, so that we refrained from observing flux standards.

The radial velocities of the March and April data were measured with respect to an average of several night-sky lines, by fitting single Gauss functions to the lines both from the object and from the night sky. The period search within the individual data sets were done by using the Scargle (1982) and AOV (Schwarzenberg-Czerny 1989) algorithms implemented in ESO-MIDAS. A method similar to that of Thorstensen \& Freed (1985) was used to check for alias periods (Mennickent \& Tappert 2001).

\footnotetext{
3 Asiago Faint Object Spectrograph and Camera.
}

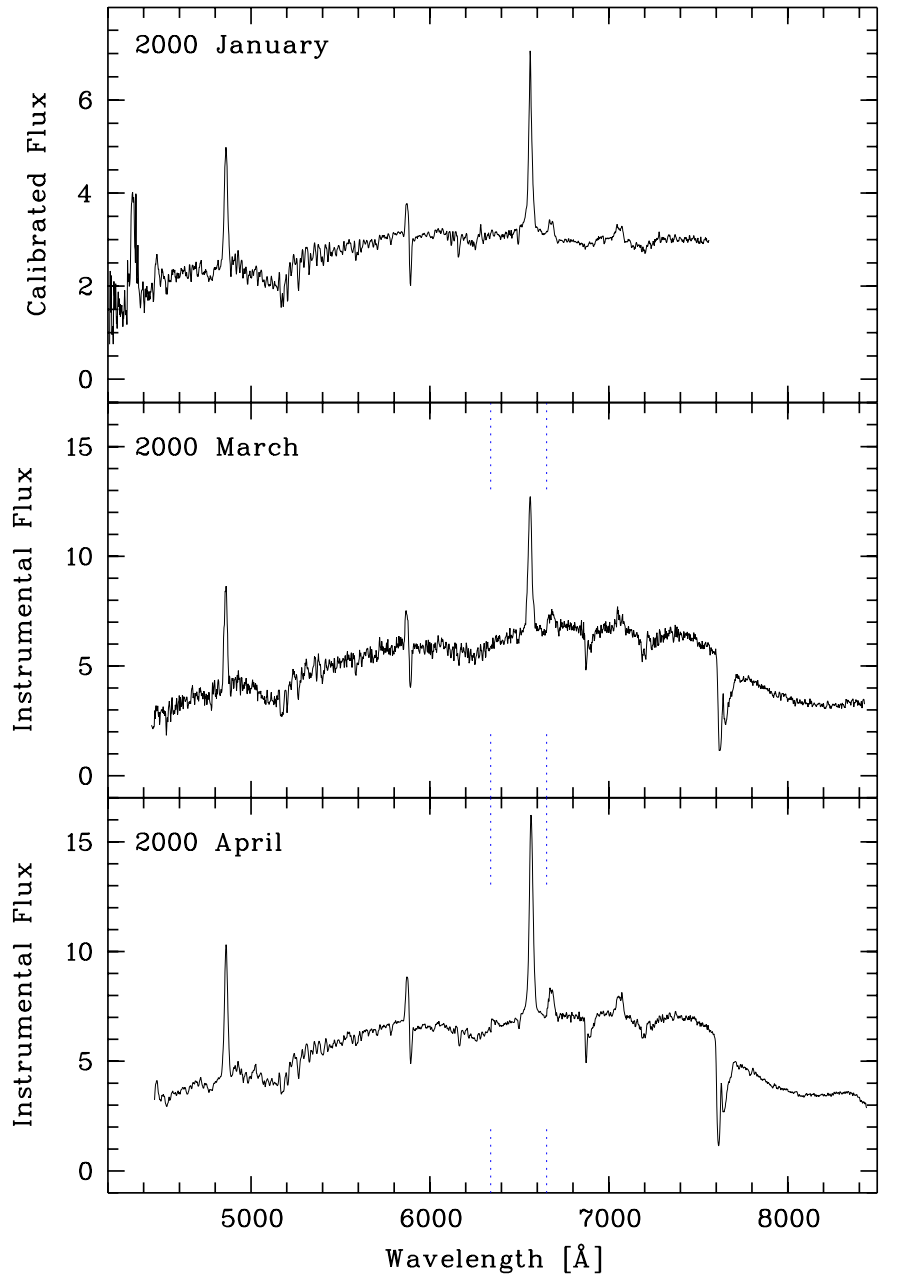

Fig. 1. Average spectra of CW1045+525 from January (top), March (middle), and April (bottom), 2000. The dotted lines in the lower plots mark the overlapping region of grism \#7 and grism \#8. The flux of the upper plot is in $10^{-15} \mathrm{erg} \mathrm{cm}^{-2} \mathrm{~s}^{-1} \AA^{-1}$, the instrumental flux of the two lower ones has been divided by 100 for presentation purposes. Note that those spectra have not been corrected for the instrumental response curve.

The instrumental magnitudes of the photometric data were extracted with respect to an average lightcurve composed out of several comparison stars. Three of these stars were included in the atlas of secondary photometric standards by Henden \& Honeycutt (1995), enabling the computation of calibrated magnitudes for CW 1045+525 within an error of $\sim 0.02$ mag.

Table 1 summarizes the data on the observations.

\section{Results}

\subsection{Spectrum and orbital period}

\subsubsection{Properties of the average spectrum}

The averaged spectra for January, March, and April 2000, are shown in Fig. 1. In the case of the latter two, first the individual spectra of grism \#7 and of grism \#8 were averaged. The resulting spectra were rebinned to the 
Table 1. Overview on the observations. $n_{\text {data }}$ gives the number of data points, $t_{\exp }$ the individual exposure time, $\Delta t$ the time range covered by the observations, $\Delta \lambda$ the $F W H M$ resolution, and $\Delta V(\Delta B)$ the magnitude range of the object during the night in $V(B)$. For the $2.4 \mathrm{~m}$ Hiltner observations, $\Delta \lambda$ was measured by fits to the night sky line, the corresponding data for the $1.82 \mathrm{~m}$ Ekar refer to fits to the calibration spectra. The magnitudes for the Hoher List and the Ekar data have been derived by differential photometry and comparison to secondary photometric standards, and should be accurate within the cited errors.

\begin{tabular}{|c|c|c|c|c|c|c|c|}
\hline date & telescope & $n_{\text {data }}$ & $t_{\exp }[\mathrm{s}]$ & $\Delta t[\mathrm{~h}]$ & filter / range $[\AA]$ & $\Delta \lambda[\AA]$ & $\Delta V / \Delta B[\mathrm{mag}]$ \\
\hline 1998-03-12 & $1 \mathrm{~m}$ Hoher List & 92 & 90 & 2.78 & $V$ & - & $15.399(35)-15.191(53)$ \\
\hline 1999-03-10 & $1 \mathrm{~m}$ Hoher List & 150 & 120 & 6.74 & $V$ & - & $15.439(21)-15.165(20)$ \\
\hline 1999-03-11 & $1 \mathrm{~m}$ Hoher List & 191 & 120 & 8.28 & $V$ & - & $15.408(23)-15.109(17)$ \\
\hline 1999-03-12 & $1 \mathrm{~m}$ Hoher List & 18 & 120 & 1.39 & $V$ & - & $15.327(29)-15.108(73)$ \\
\hline 1999-03-13 & $1 \mathrm{~m}$ Hoher List & 58 & 120 & 6.72 & $V$ & - & $15.62(14)-15.02(23)$ \\
\hline 1999-03-14 & $1 \mathrm{~m}$ Hoher List & 163 & 120 & 8.47 & $V$ & - & $15.358(22)-15.094(20)$ \\
\hline 2000-01-07 & 2.4 m Hiltner & 6 & 360 & 2.61 & $4210-7560$ & 3.6 & \\
\hline 2000-01-08 & $2.4 \mathrm{~m}$ Hiltner & 5 & 360 & 7.08 & $4210-7560$ & 3.6 & \\
\hline 2000-01-09 & $2.4 \mathrm{~m}$ Hiltner & 3 & 360 & 5.86 & $4210-7560$ & 3.6 & \\
\hline 2000-01-10 & $2.4 \mathrm{~m}$ Hiltner & 7 & 360 & 0.65 & $4210-7560$ & 3.6 & \\
\hline 2000-03-02 & $1.82 \mathrm{~m} \mathrm{Ekar}$ & 2 & 60 & 0.26 & B & - & $16.005(26)-15.997(26)$ \\
\hline 2000-03-02 & $1.82 \mathrm{~m} \mathrm{Ekar}$ & 17 & 60 & 2.50 & $V$ & - & $15.197(19)-15.083(19)$ \\
\hline 2000-03-02 & $1.82 \mathrm{~m} \mathrm{Ekar}$ & 2 & 60 & 0.24 & $R$ & - & \\
\hline 2000-03-02 & $1.82 \mathrm{~m} \mathrm{Ekar}$ & 1 & 300 & - & $4444-6677$ & 9.2 & \\
\hline 2000-03-03 & $1.82 \mathrm{~m} \mathrm{Ekar}$ & 8 & 60 & 5.50 & B & - & $15.955(26)-15.685(26)$ \\
\hline 2000-03-03 & $1.82 \mathrm{~m} \mathrm{Ekar}$ & 9 & 30 & 5.50 & $V$ & - & $15.163(19)-14.945(20)$ \\
\hline 2000-03-03 & $1.82 \mathrm{~m} \mathrm{Ekar}$ & 9 & 30 & 5.47 & $R$ & - & \\
\hline 2000-03-03 & $1.82 \mathrm{~m} \mathrm{Ekar}$ & 18 & 600 & 6.39 & $6328-8430$ & 8.4 & \\
\hline 2000-04-07 & $1.82 \mathrm{~m} \mathrm{Ekar}$ & 7 & 20 & 6.79 & $V$ & - & $15.156(28)-15.008(26)$ \\
\hline 2000-04-07 & $1.82 \mathrm{~m} \mathrm{Ekar}$ & 16 & 300 & 7.21 & $4460-6660$ & 9.2 & \\
\hline 2000-04-07 & $1.82 \mathrm{~m} \mathrm{Ekar}$ & 13 & 300 & 7.17 & $6340-8442$ & 8.4 & \\
\hline 2001-03-26 & $2.4 \mathrm{~m}$ Hiltner & 1 & 480 & - & $4210-7560$ & 3.8 & \\
\hline 2001-03-27 & $2.4 \mathrm{~m}$ Hiltner & 1 & 480 & - & $4210-7560$ & 3.8 & \\
\hline 2001-05-12 & $2.4 \mathrm{~m}$ Hiltner & 2 & 480 & 1.64 & $4210-7560$ & 5.9 & \\
\hline 2001-05-14 & $2.4 \mathrm{~m}$ Hiltner & 1 & 480 & - & $4210-7560$ & 3.7 & \\
\hline 2001-05-17 & $2.4 \mathrm{~m}$ Hiltner & 1 & 480 & - & $4210-7560$ & 3.7 & \\
\hline
\end{tabular}

dispersion of grism \#8, and, after ensuring that the overlapping regions would match, were then combined to yield a single average spectrum over the whole spectral range. Although these spectra are not flux-calibrated, the comparison with the Kitt Peak data shows that the instrumental response curve affects the continuum shape only weakly.

Table 2 shows the properties of the principle spectral features of the average spectra, i.e. of the Balmer and $\mathrm{He}$ I emission, and of the NaI absorption. The total absence of high ionized emission lines like He II $\lambda 4686$ is noteworthy.

Comparing the average spectra for each month shows that while the March and January data have very similar values, the equivalent widths $W_{\lambda}$ of all emission lines in the April data are significantly higher. However, the calibrated magnitudes in Table 1 show no corresponding behaviour for the continuum for the March and April data (the uncertainties of spectrophotometrically derived magnitudes of the January data impede a conclusive comparison). It appears that the difference is due to an enhancement of the line-emission region only.

In order to check if this phenomenon corresponds to real long-term behaviour, or if it is due to orbital changes and average spectra composed from data not uniformly distributed in phase, we measured $W_{\lambda}$ for the $\mathrm{H} \alpha$ emission line of the individual spectra and folded it according to the ephemeris derived below. The result in Fig. 2 clearly shows a) that there is no convincing phase-dependent variation of $W_{\lambda}$, and $\mathrm{b}$ ) that on average there are significant differences for the individual data sets, probably reflecting changes in the mass-transfer rate. This behaviour is also evident in the traced spectrum of the $\mathrm{H} \alpha$ line (Fig. 5).

The spectrum of CW $1045+525$ (Fig. 1) shows a strong late-type contribution, similar to previously-published spectra. We estimated the secondary's contribution and spectral type using K dwarf spectra from Pickles' (1998) library. We scaled these with a range of factors, and 
Table 2. Properties of the principle spectral features of the average spectra. Negative equivalent widths $W_{\lambda}$ denote emission lines. Colons mark uncertain data due to noise or blends.

\begin{tabular}{|c|c|c|c|c|}
\hline$\lambda[\AA]$ & ID & $\lambda_{\text {rest }}$ & $W_{\lambda}[\AA]$ & $F W H M[\AA]$ \\
\hline \multicolumn{5}{|c|}{2000 January: } \\
\hline 4340 & $\mathrm{H} \gamma$ & 4341 & -43 & 25: \\
\hline 4473 & He I & 4471 & -7 & 19: \\
\hline 4860 & $\mathrm{H} \beta$ & 4861 & -25 & 21 \\
\hline 5871 & $\mathrm{He} \mathrm{I}$ & 5876 & $-5:$ & 17: \\
\hline 5892 & $\mathrm{NaI} D$ & $5890 / 5896$ & 4: & 11: \\
\hline 6561 & $\mathrm{H} \alpha$ & 6563 & -26 & 22 \\
\hline 6678 & $\mathrm{He} \mathrm{I}$ & 6678 & -4 & 30 \\
\hline 7058 & $\mathrm{HeI}$ & 7065 & -4 & 45: \\
\hline \multicolumn{5}{|c|}{2000 March: } \\
\hline 4859 & $\mathrm{H} \beta$ & 4861 & -22 & 20 \\
\hline 5869 & $\mathrm{HeI}$ & 5876 & $-5:$ & 15: \\
\hline 5892 & $\mathrm{NaI} D$ & $5890 / 5896$ & 4: & 12: \\
\hline 6559 & $\mathrm{H} \alpha$ & 6563 & -24 & 25 \\
\hline 6682 & He I & 6678 & -5 & 40 \\
\hline 7056 & $\mathrm{HeI}$ & 7065 & -4 & 44 \\
\hline \multicolumn{5}{|c|}{2000 April: } \\
\hline 4473 & He I & 4471 & $-5:$ & 15: \\
\hline 4861 & $\mathrm{H} \beta$ & 4861 & -33 & 22 \\
\hline 4928 & $\mathrm{He} \mathrm{I}$ & 4922 & $-6:$ & 11: \\
\hline 5022 & He I & 5016 & $-3:$ & $27:$ \\
\hline 5872 & $\mathrm{He} \mathrm{I}$ & 5876 & $-6:$ & 17: \\
\hline 5894 & $\mathrm{NaI} D$ & $5890 / 5896$ & 3: & 11: \\
\hline 6566 & $\mathrm{H} \alpha$ & 6563 & -34 & 24 \\
\hline 6678 & He I & 6678 & -6 & 35 \\
\hline 7059 & $\mathrm{HeI}$ & 7065 & -7 & 43 \\
\hline
\end{tabular}

subtracted them from a version of our mean spectrum which had been smoothed to match the somewhat lower resolution of the library spectra. Acceptable cancellation of the late-type features (especially the broad absorption feature centered near $\lambda 5180)$ could be obtained for stars of type K5 through M0, but only if the subtracted signal constituted most of the light in the observed range. If we assume the same brightness level of the system during all spectroscopic observations, we obtain a mean magnitude for the average Januray spectrum of $V \sim 15.1$; in the best deconvolution, about 70 per cent of the flux is from the K-star, yielding an average $V \sim 15.5$ for the $\mathrm{K}$ star alone.

\subsubsection{Radial velocities and the orbital period}

For the MDM data, we measured radial velocities of the late-type spectrum using cross-correlation techniques. For the template spectrum we used a velocity-compensated sum of spectra of G- and K-type IAU velocity standards. To avoid emission lines only the region $5020<\lambda<$ $5800 \AA$ was used. The correlation peaks were very strong

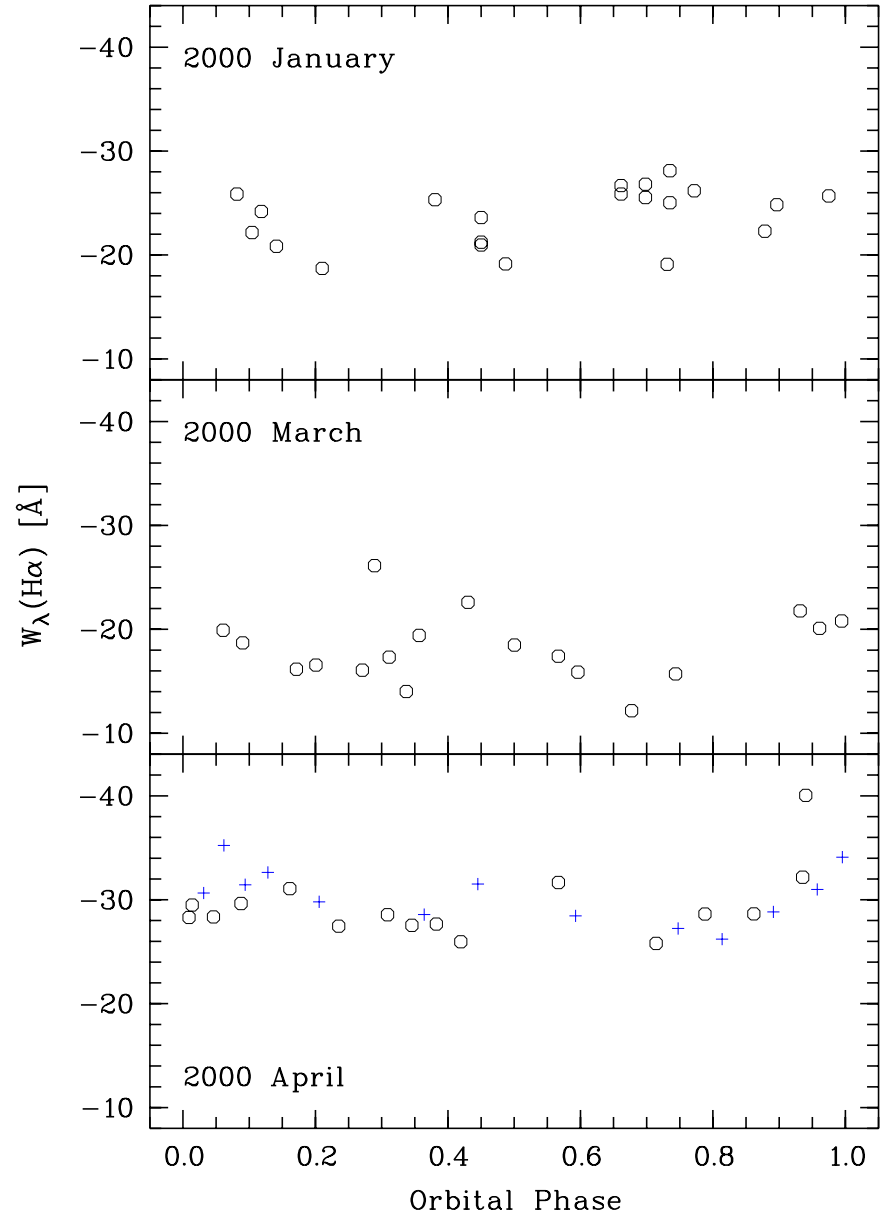

Fig. 2. Equivalent widths of the $\mathrm{H} \alpha$ emission line from January (top), March (middle), and April (bottom), data. In the latter plot, the different symbols denote measurements with grism \#7 $(+)$ and grism \#8 (O).

and the estimated statistical uncertainties were generally $<10 \mathrm{~km} \mathrm{~s}^{-1}$. The $\mathrm{H} \alpha$ emission line was measured using a correlation function consisting of positive and negative Gaussians, each with FWHM $8 \AA$ and separated by $30 \AA$. A period near $0.27 \mathrm{~d}$ was plainly apparent in both the absorption and emission-line velocities. The Ekar absorption velocities (2000 April) are less precise than those from MDM but they do show the modulation, and help constrain the ephemeris. They were included in the period search analysis, with a $-63 \mathrm{kms}^{-1}$ zero point offset to bring them in line with the MDM velocities.

Figure 3 shows a period search of the combined absorption velocity data, which span $495 \mathrm{~d}$ of elapsed time. Because the uncertainties in the velocities are much smaller than their modulation, a single frequency corresponding to $P_{\text {orb }}=0.271278 \mathrm{~d}$ is selected without any cycle-count ambiguity. Figure 4 shows the emission and absorption velocities folded on this period, with the best fitting sinusoids superposed. The blue-to-red crossing of the absorption velocities follow the ephemeris

$T_{0}=$ HJD 2451642.621(1) $+0.271278(1) E$, 


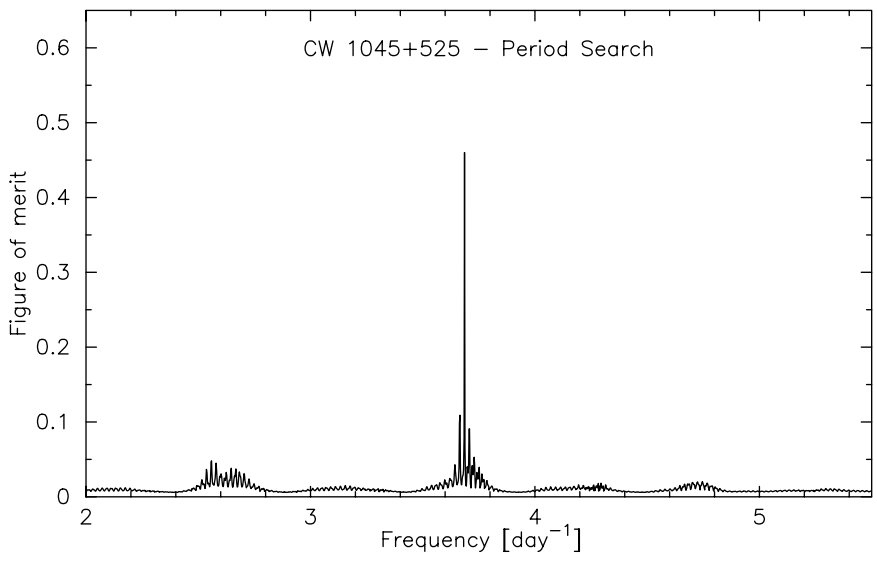

Fig. 3. Period search of the absorption-line radial velocities in the vicinity of the best fit. The figure of merit is $1 . /\left[\sum_{i}\left(\left(o_{i}-\right.\right.\right.$ $\left.\left.\left.c_{i}\right) / \sigma_{i}\right)^{2}\right]$, where $o_{i}$ are the observed velocities, $c_{i}$ the velocities computed from the best-fitting sinusoid at each trial frequency, and $\sigma_{i}$ are the estimated velocity uncertainties.

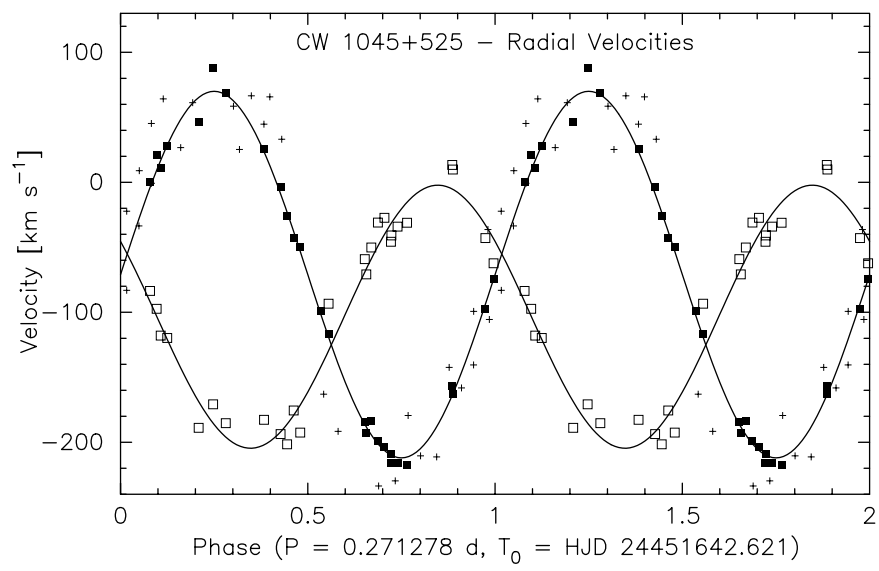

Fig. 4. Radial velocity data folded with $P=0.271278 \mathrm{~d}$ with respect to the zero point (inferior conjunction) of the MDM absorption spectra (filled squares). The small crosses show the 2000 April Ekar absorption velocities, offset by $-63 \mathrm{~km} \mathrm{~s}^{-1}$ to match the MDM zero point. $\mathrm{H} \alpha$ emission velocities are shown by open squares. Also shown are the best sinuoids for both components. Note the evident phase shift $\Delta \varphi=0.595(10)$ cycles of the sinusoids.

where $E$ is an integer. The parameters of the best-fit sinusoids are given in Table 3.

The absorption-line velocities should rather faithfully reflect the phase of the secondary star's motion (though the amplitude may be affected by illuminated-atmosphere and other effects; Wade \& Horne 1988). If the emission-line velocities were to follow the motion of the white dwarf, the phase of their motion should be $1 / 2$ cycle from the absorption line phase. It is therefore sobering that the emission and absorption line phases differ by significantly more, specifically $0.595 \pm 0.010$ cycles. Furthermore, the mean velocities $\gamma$ differ significantly. These differences are clear in Fig. 4. This underscores the dangers of using emission lines for dynamical purposes - while they reliably indicate the period, they may not reflect the center-of-mass motion of either star (e.g., Tappert 1999).

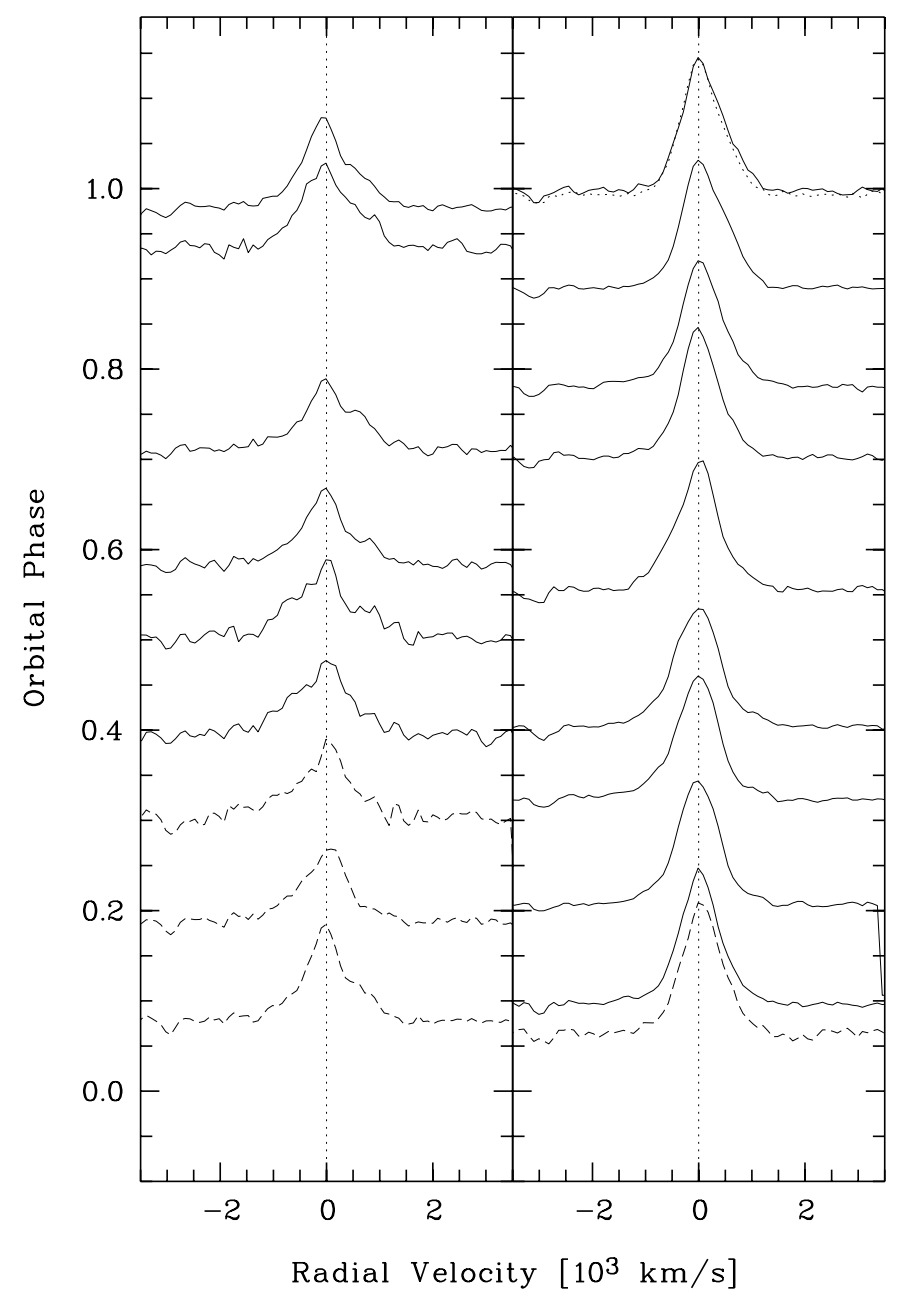

Fig. 5. Traced spectrum of the $\mathrm{H} \alpha$ emission line in the Ekar spectra (right: 2000-03-03, left: 2000-04-07; grism \#8 only), subsequently binned into $10 \%$ phase bins. The time sequence is symbolized by the line styles dotted - solid - dashed. Zero phase refers to the ephemeris (1). Note the asymmetric line profile especially evident in phases 0.6 and 1.0.

\subsubsection{Line profile analysis}

The differences in phase between the absorption and the emission lines found in the last section indicate the presence of an isolated emission source, i.e. an emission region which is not distributed symmetrically around the white dwarf. A distribution of the latter type, e.g. an emission ring within the accretion disk, would result in a symmetric line profile, with orbital variations only in position, but not in shape (e.g., Horne \& Marsh 1986, and references therein). In the case of CW $1045+525$, however, the line profile of $\mathrm{H} \alpha$ is clearly asymmetric at certain phases (Fig. 5).

In order to obtain a quantitative description of this behaviour, one can compare the flux in the blue half $F(V)$ of the line profile to the one in the red half $F(R)$ by defining (Tappert 1999)

$V / R=\log \frac{F(V)}{F(R)}$ 
Table 3. Parameters of the sine fits to the MDM radial velocities. Fits are of the form $v(t)=\gamma+K \sin \left[2 \pi\left(t-T_{0}\right) / P\right]$. For the fit to the $\mathrm{H} \alpha$ velocities, $P$ was held fixed.

\begin{tabular}{llllll}
\hline data set & $T_{0}[\mathrm{HJD}]$ & $P[\mathrm{~d}]$ & $K\left[\mathrm{~km} \mathrm{~s}^{-1}\right]$ & $\gamma\left[\mathrm{km} \mathrm{s}^{-1}\right]$ & $\sigma\left[\mathrm{km} \mathrm{s}^{-1}\right]$ \\
\hline Absn. & $2451553.913(1)$ & $0.271278(1)$ & $141(3)$ & $-71(2)$ & 7 \\
$\mathrm{H} \alpha$ & $2451553.804(2)$ & {$[0.271278]$} & $101(5)$ & $-103(3)$ & 14 \\
\hline
\end{tabular}

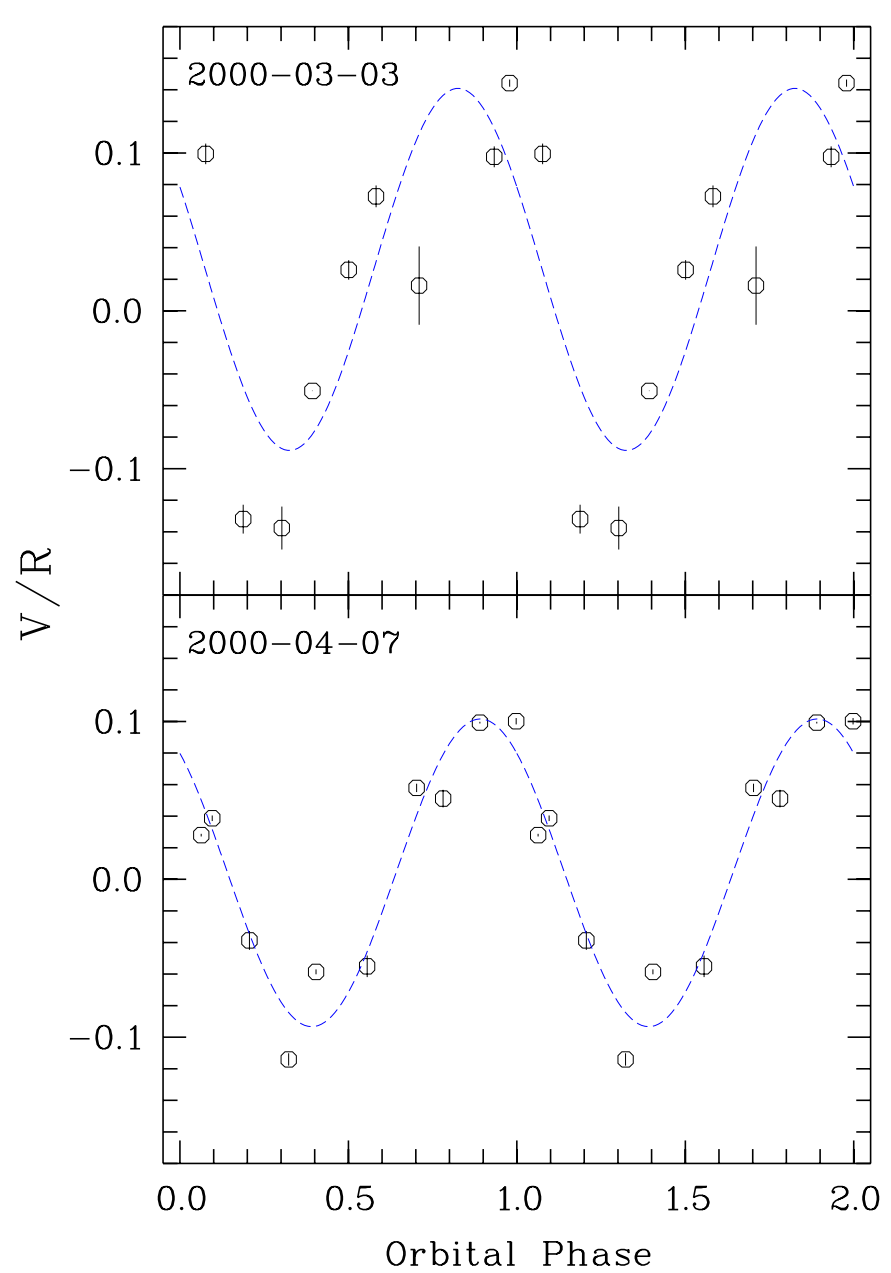

Fig. 6. $V / R$ plot of the $\mathrm{H} \alpha$ line from the March (top) and the April (bottom) data. The dashed curves give the best sine fits to the individual data sets.

The point which separates both halves is here chosen as the centre of the line flanks at three intensity values, specifically at $1 / 4,1 / 5$, and $1 / 6$, of the maximum intensity. This proved to be sufficiently above the noisedominated extreme line wings, as well as far enough below the peak to consider the main part of the line. The actual $V / R$ is then taken as the average of the three values, its sigma being indicative of the noise in the line. A more detailed description of this method will be given elsewhere (Tappert et al., in preparation).

The resulting $V / R(\varphi)$ were fitted with a sine function

$V / R(\varphi)=\gamma-K \sin (2 \pi \varphi)$.

The derived parameters are listed in Table 4, the actual fits and the data are plotted in Fig. 6. The relative amplitude error $\sigma(K) / K$ is a measure of the significance of the variation. As a comparison, a corresponding examination of a symmetric, undisturbed, line profile gave $\sigma(K) / K=0.66$ (Tappert et al. in preparation). Therefore, CW $1045+525$ shows a significant variation in both data sets. The sigma in March is higher than in April, due to the lower $S / N$ of the data. Nevertheless, both data sets show basically identical variations, with zero points of the negative-to-positive crossing at $\varphi_{0} \sim 0.6$. This point corresponds to the superior conjunction of the additional emission source (the asymmetric emission changes from the red to the blue half $)^{4}$. The obtained location in our data is thus a rather unusual one. Emission from the bright spot region or from the secondary, e.g., would have values of $\varphi_{0} \sim 0.35$ and 0.5 , respectively.

The spectrum in Fig. 5 shows, furthermore, that the line peak has rather low radial velocity and stays close to the dotted line which marks zero radial velocity, while the line wings seem to have higher values. To investigate this in more detail, we computed a diagnostic diagram (Shafter 1983). This method shows the parameters of the radial-velocity curve as a function of the separation of the two Gaussians used for the velocity determination (see Sect. 3.1.2). Figure 7 shows the result of using Gaussians with $F W H M=4 \AA$ with the April data. The fact that the semi-amplitude $K_{1}$ increases with the separation confirms our suspicion based on the visual impression. It appears thus that the line profile of CW $1045+525$ consists of a low-velocity, almost stationary, central component and "high-velocity" 5 wings. With this finding, the precise phasing of the $V / R$ variation loses importance, as it is not mainly caused by the movement of an additional emission (like e.g. in U Gem; Stover 1981), but rather by the movement of the line wings around the (almost) stationary component, explaining the phase shift of roughly 0.5 orbits between the $V / R$ zero phase and the line wings.

\subsection{Lightcurve}

Of all the photometric data, only the measurements at the Hoher List from 1999-03-10, 11, and 14, have both sufficient time resolution and coverage to define the shape of

\footnotetext{
${ }^{4}$ Note that the corresponding radial-velocity curve is expected to be antiphased to the $V / R$ curve. This is not the case for the data presented here, as our radial-velocity measurements refer to the wings of the line profile.

${ }^{5}$ We here use quotations marks, as these velocities stay rather within the range of the usually observed values for CVs, and to distinguish them from the high-velocity components observed in certain nova-likes (e.g., Taylor et al. 1999, for LS Peg).
} 


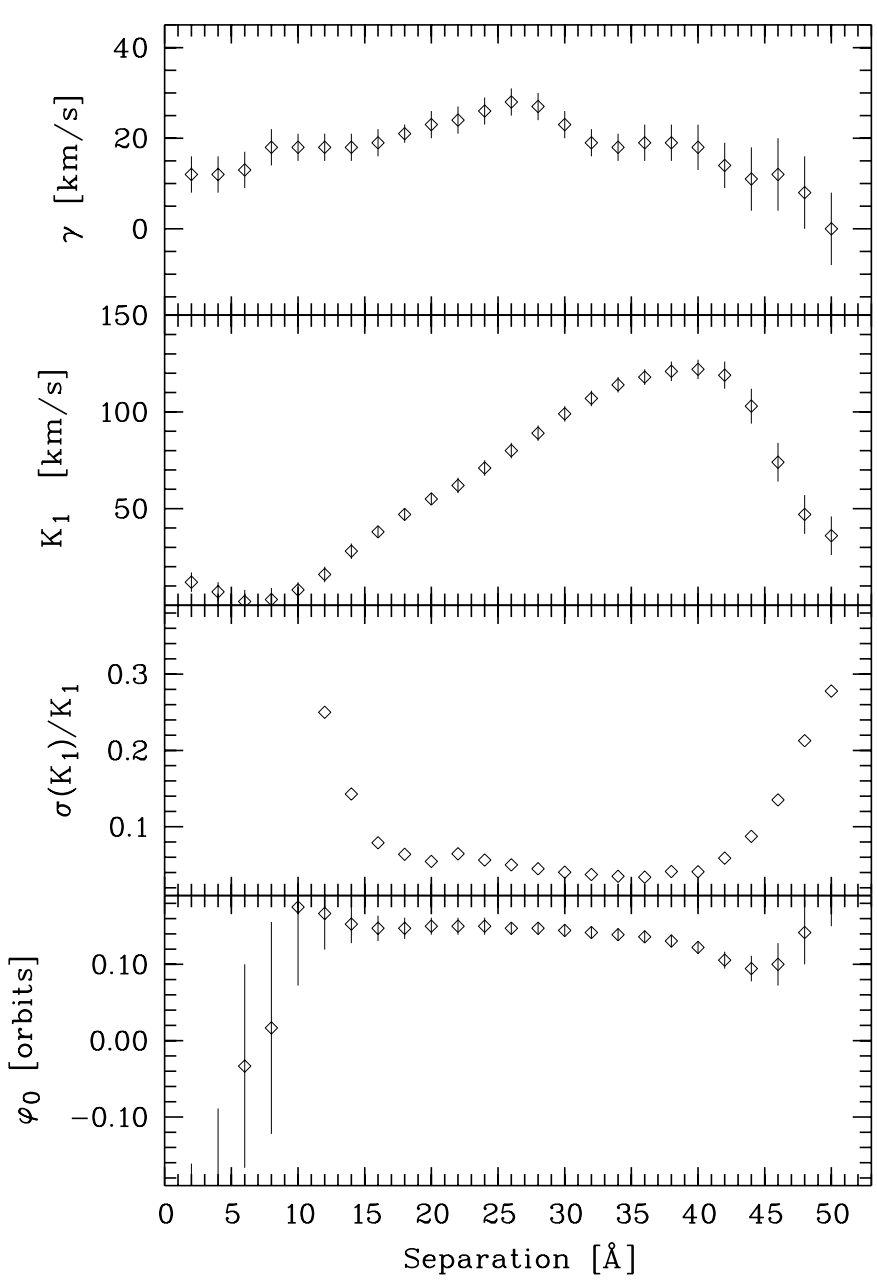

Fig. 7. Diagnostic diagram of the 2000-04-07 data, showing the parameters of the radial-velocity fit $v_{\mathrm{r}}(\varphi)=\gamma-K_{1} \sin (2 \pi \varphi)$ as functions of the Gaussian separation. The zero point of the orbital phase, $\varphi_{0}=0.0$, refers to ephemeris (1). Note that this point is not reached for separations which correspond to data not dominated by noise (separations $d<44 \AA$ ).

Table 4. Parameters of the sine fits to the $V / R$ values of the $\mathrm{H} \alpha$ line. The zero point $\varphi_{0}$ refers to the negative-positive crossing of the fit and corresponds to the superior conjunction of the emission source.

\begin{tabular}{lllll}
\hline data set & $\gamma$ & $K$ & $\varphi_{0}$ & $\sigma(K) / K$ \\
\hline $03-03$ & $0.026(13)$ & $0.115(21)$ & $0.575(28)$ & 0.183 \\
$04-07$ & $0.004(05)$ & $0.097(07)$ & $0.641(14)$ & 0.067 \\
\hline
\end{tabular}

the lightcurve. These individual data sets are presented in Fig. 8. Each set covers a time range larger than the orbital period (Table 1). We note that both in the middle and the lower plot the distance between the two deep minima amounts to exactly one orbital cycle. In the upper data set, this time range roughly corresponds to the first, shallow, minimum and the end of the data set. As the individual lightcurves are furthermore of very similar appearance, it seems safe to assume that the observed variation with $\Delta V \sim 0.3 \mathrm{mag}$ is modulated with the orbital period.

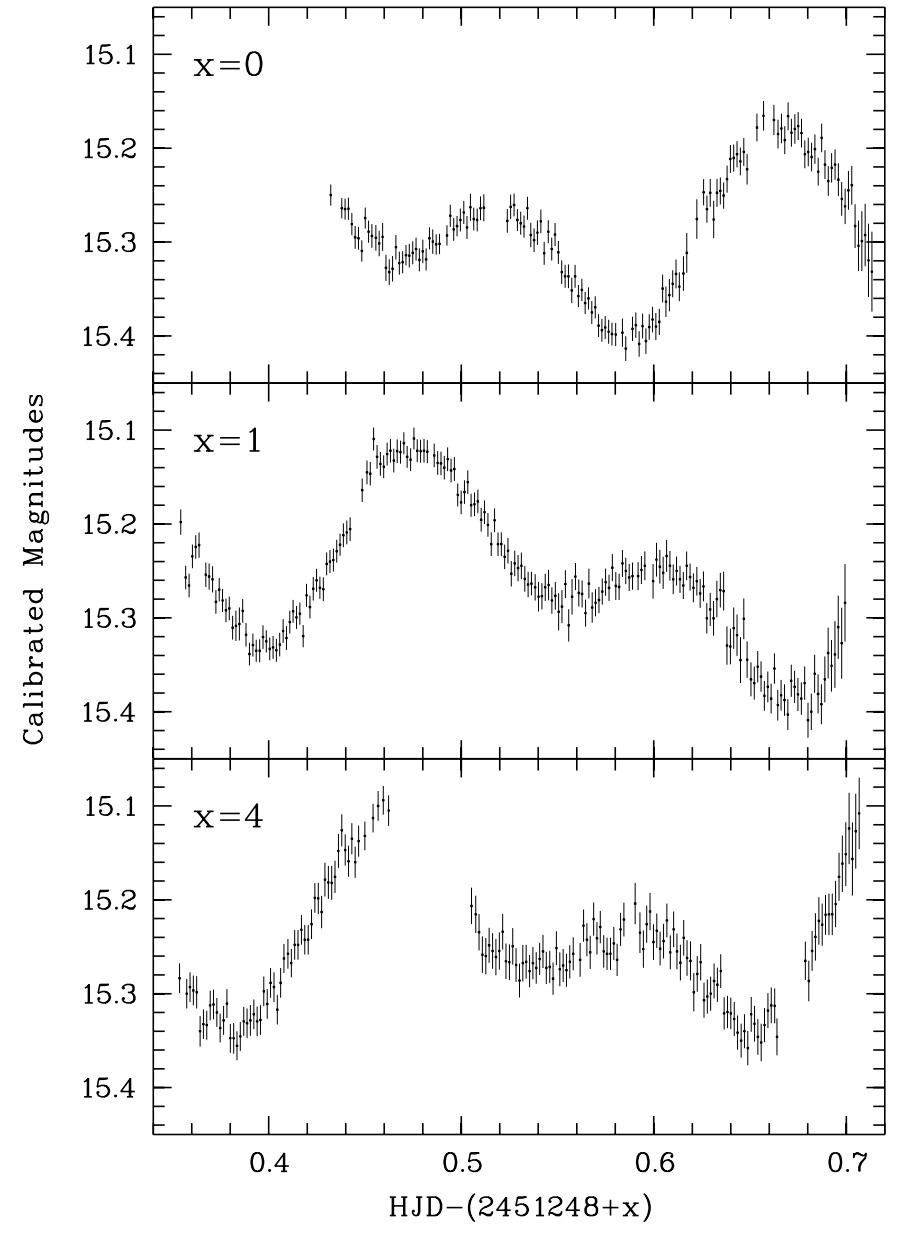

Fig. 8. Individual lightcurves from 1999-03-10 (top), 11 (middle), and 14 (bottom).

Apart from this variation, which will be discussed below, the plot sequence also indicates some minor variations at non-orbital time-scales, especially evident in the data from 1999-03-11 (middle plot in Fig. 8) which show a linear decline of $\sim 0.3 \mathrm{mag} / \mathrm{d}$. We furthermore note that all lightcurves are remarkably smooth and do not show any clear evidence for flickering, usually the signature of an accretion disc in CVs. This behaviour, together with the distinctive shape of the lightcurve, is very reminiscent of a magnetic CV in a low state (e.g., Burwitz et al. 1998). We discuss the pro and cons of this possibility in Sect. 5.2.

The spectroscopically derived ephemeris (1) allows an extrapolation to the photometric measurements with a precision of 0.005 orbital phases. In order to establish an average lightcurve, we normalized the data by subtracting the mean value in the case of 1999-03-10 and 14, and by subtracting a linear function with a slope of $0.31 \mathrm{mag} / \mathrm{d}$ for 1999-03-11 to account for the decline. After applying some additional minor magnitude shifts, we then averaged the data in phase bins of 0.005 orbits. The resulting lightcurves are shown in Fig. 9.

The shape of the lightcurve consists of two maxima and two minima of unequal height and depth, respectively. While the double-hump structure can be explained 


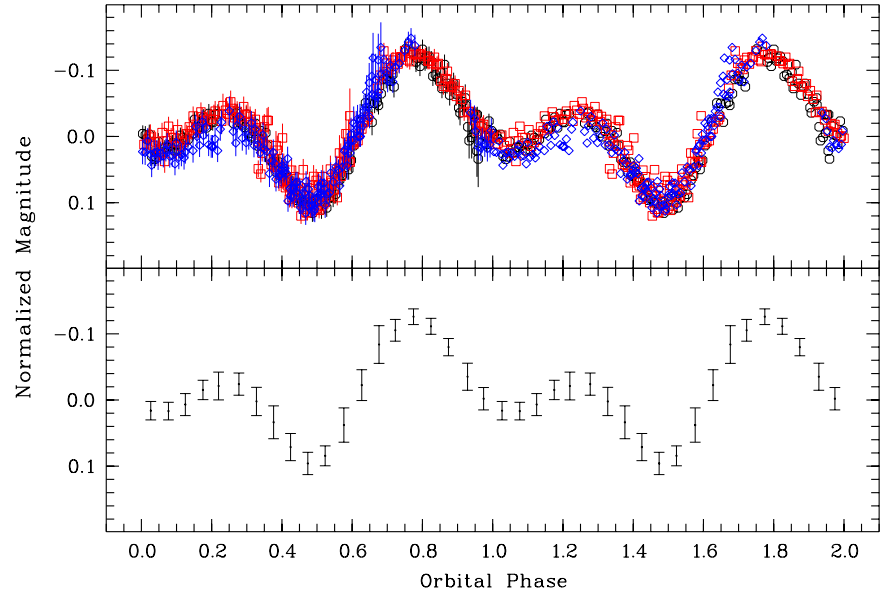

Fig. 9. Orbital lightcurve of CW 1045+525. Top: combined normalized data from 1999-03-10 (O), $11(\square)$, and $14(\diamond)$. A linear decline with a slope of $0.31 \mathrm{mag} / \mathrm{d}$ has been subtracted from the 1999-03-11 data. For presentation purposes, two orbital phases are shown, the second one without error bars. Bottom: the data from the upper plot averaged into 0.05 phase bins. The error bars indicate the sigma of the averaging process.

Table 5. Description of the features in the average lightcurve and their possible explanation. The phase error was estimated on the basis of several measurements with a graphics cursor.

\begin{tabular}{|c|c|c|}
\hline feature & phase & explanation \\
\hline shallow minimum & $0.05(1)$ & $\begin{array}{l}\text { inferior conjunction of the } \\
\text { secondary, minimum surface } \\
\text { brightness due to circular } \\
\text { projection, additional source } \\
\text { unobscured }\end{array}$ \\
\hline small maximum & $0.24(1)$ & $\begin{array}{l}\text { secondary at } 90^{\circ} \text {, maximum } \\
\text { surface brightness due to } \\
\text { maximally projected elon- } \\
\text { gation, additional source } \\
\text { (partly) obscured }\end{array}$ \\
\hline deep minimum & $0.49(1)$ & $\begin{array}{l}\text { superior conjunction of the } \\
\text { secondary, minimum surface } \\
\text { brightness due to circular } \\
\text { projection, additional source } \\
\text { (partly) obscured }\end{array}$ \\
\hline large maximum & $0.77(1)$ & $\begin{array}{l}\text { secondary at } 270^{\circ} \text {, maxi- } \\
\text { mum surface brightness } \\
\text { due to maximally projected } \\
\text { elongation, additional source } \\
\text { unobscured }\end{array}$ \\
\hline
\end{tabular}

as being due to the ellipsoidal variation of the secondary, the different size of the features indicates the presence of a second light source in the system. The characteristics of the average lightcurve and their possible explanations are described in Table 5.

In order to test if our interpretation of the lightcurve is consistent with the analysis of the spectroscopic data, a double sine function was subtracted from the normalized combined data. The sine was shifted in phase by precisely 0.25 orbits to correspond to the theoretical phasing

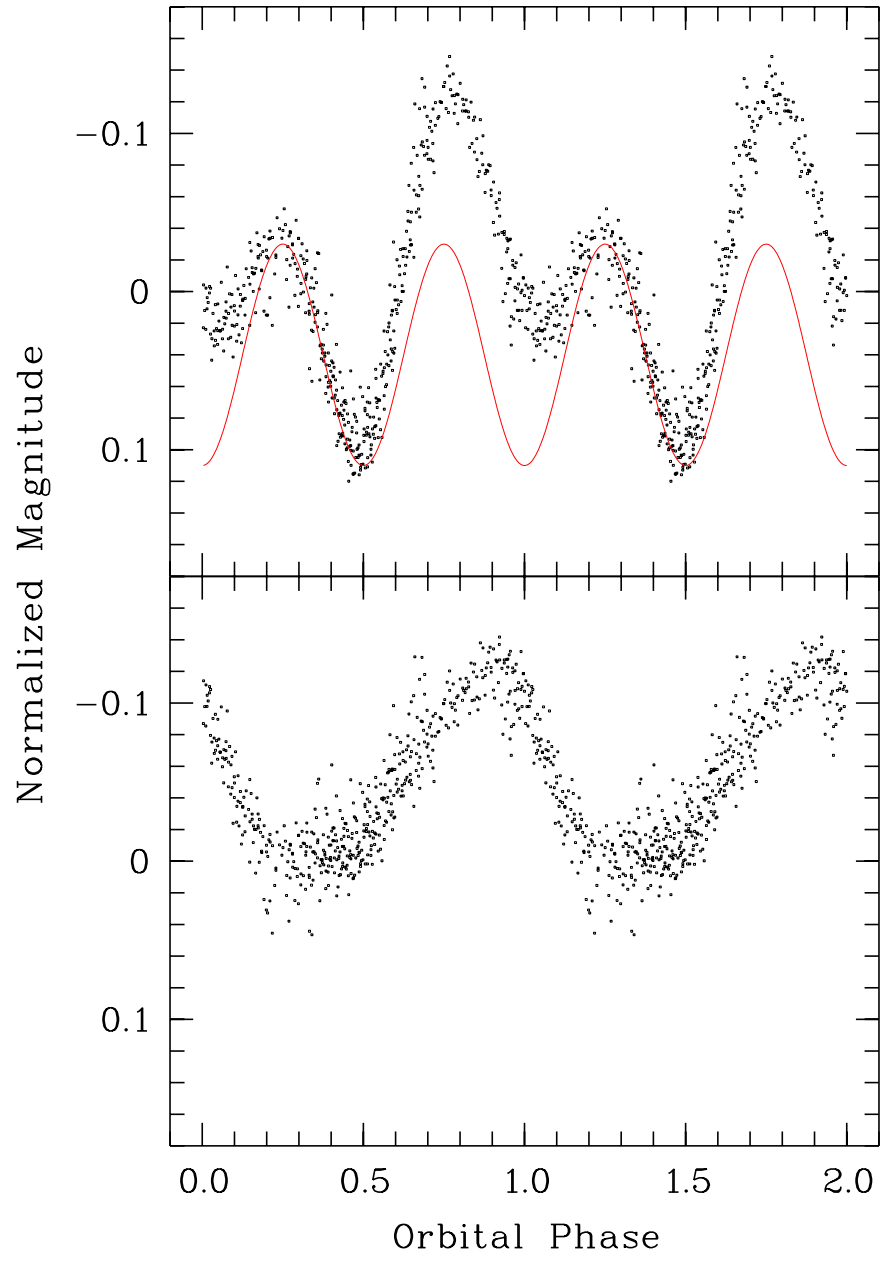

Fig. 10. The combined lightcurve, together with a sinusoidal function shifted by 0.25 orbital phases and adjusted in zero point and amplitude to match the data (top), and the sinesubtracted data (bottom).

of the secondary's ellipsoidal variation, and shifted in magnitude by +0.04 mag from the zero point of the normalized lightcurve. The latter shift and the amplitude of 0.07 mag were manually adjusted to match the shallow maximum and the deep minimum. Following our interpretation, these two features should be least influenced by the additional light source. This is supported by the fact that they represent the only "orbital symmetric" features, i.e. their phase difference amounts to $1 / 4$ of the orbital cycle.

The residual lightcurve of this subtraction (Fig. 10) shows an asymmetric hump with a slow rise to a maximum at phase 0.92, and a following steeper decline. All this is consistent with a feature produced by a typical bright spot. Our rather simple approach does not really prove that this picture is correct or represents the only possible explanation, but it indeed agrees well with the spectroscopically derived values (the zero phase of the orbital motion and the precision of the orbital period). We return to the discussion of the lightcurve in Sect. 5 . 


\section{Dynamics and distance}

The orbital period and the spectral type of the secondary allow us to constrain the distance and luminosity of the system. Beuermann et al. (1999) tabulate absolute magnitudes, colours, and estimated radii of a sample of nearby $\mathrm{K}$ and $\mathrm{M}$ dwarfs (their Table 3). Scaling these data, we find that hypothetical $1 R_{\odot}$ stars of type $\mathrm{K} 4$ and type M0 should respectively have $M_{V}=+6$ and +7.2 . The orbital period and Roche constraints (Beuermann et al. 1998, Eq. (1)) yield

$$
\left(R_{2} / R_{\odot}\right)=f(q) 0.82\left(M_{2} / M_{\odot}\right)^{(1 / 3)}
$$

at $P=0.272 \mathrm{~d}$, where the subscript 2 refers to the secondary star, and the function $f(q)$ is within 3 percent of unity for $q=M_{2} / M_{1} \leq 1$. Because the secondary is likely to be modified by mass transfer (see, e.g., Beuermann et al. 1998), we do not assume a main-sequence massradius relation, but rather use the evolutionary models calculated by Baraffe \& Kolb (2000) as an approximate guide. Interpolating their models to $6.5 \mathrm{~h}$, we find a range of masses from 0.27 to $0.81 M_{\odot}$, but the lowest-mass models are estimated to have spectral types near M2, which is later than we observe here; a more realistic range for the CW $1045+525$ secondary appears to be $0.45-0.81 M_{\odot}$. This mass range corresponds to $0.76<R_{2} / R_{\odot}<0.93$, which in turn corresponds to stars $0.4 \pm 0.2 \mathrm{mag}$ fainter than otherwise identical stars with $R=R_{\odot}$. Putting all this together and propogating the errors, we find the secondary star to have $M_{V}=7.0 \pm 0.7$, where the error is dominated by uncertainty in the spectral type. Taking the observed magnitude of the secondary alone as $V=15.5 \pm 0.5$ (see above) then yields $m-M=8.5 \pm 0.8$, or $d=350-700 \mathrm{pc}$. At $b=56^{\circ}$, extinction is unlikely to be important. At its best-estimate distance of 500 pc, CW $1045+525$ would lie about 400 pc from the Galactic plane.

We find $K_{\mathrm{abs}}=141 \pm 3 \mathrm{~km} \mathrm{~s}^{-1}$ from the MDM velocities. Without an eclipse to constrain the inclination, this is of somewhat limited dynamical usefulness, but it is of interest to check that the system parameters are reasonable. Assuming a white dwarf mass $M_{1}=0.7 M_{\odot}$ and an orbital inclination of $45^{\circ}$ (see below), the observed mass function implies $M_{2}=0.55 M_{\odot}$, entirely consistent with the observed spectral type. These values are essentially arbitrary but serve to demonstrate that $K_{\text {abs }}$ is not problematic.

\section{Discussion}

The data presented in the previous sections, on the spectroscopic and photometric properties, and on the longterm behaviour, of CW $1045+525$ still do not provide a final conclusion on the nature of this system, but they allow to discard or favour the following scenarios with a certain probability.

\subsection{A nova-like?}

We regard this possibility as the least probable one. The only point in favour of this model is the apparent absence of outbursts. However, nova-likes are characterized by very high mass-transfer rates, establishing a stationary, and for our case important - bright and optically thick, accretion disc (e.g., Warner 1995). This neither agrees with the spectroscopic data, which show Balmer and He lines purely in emission, nor with the photometric lightcurve, which is clearly dominated by the secondary.

There remains the possibility of a nova-like in a prolonged low state, classifying the system as a $\mathrm{CV}$ of the VY Scl type, but these stars appear to be strongly confined to orbital periods of 3-4 h (e.g., Ritter \& Kolb 1998), with the sole exception of VY Scl itself $\left(P_{\text {orb }}=5.6 \mathrm{~h}\right.$; Martínez-Pais et al. 2000).

\subsection{A magnetic CV?}

In this CV-subclass, the magnetic field of the white dwarf is strong enough to impede the formation of an accretion disc fully (in polars) or partly (in intermediate polars). Instead, the matter stream from the secondary is transferred along the field lines directly onto the pole(s) of the primary. Due to the high temperatures caused by this process the higher-ionized emission lines are usually very strong in magnetic CVs. Especially He II $\lambda 4686$ often matches $\mathrm{H} \beta$ in strength (e.g., chapters 6 and 7 in Warner 1995). In the spectrum of CW $1045+525$, however, this line is not even weakly present, and neither are any other high-ionized lines. On the other hand, the detected lines (Balmer and He I), albeit being of only medium strength, are still too strong to be produced by a magnetic CV in its low state.

Although the other properties would nicely fit into the scenario of a magnetic CV in intermediate state - no outbursts, no clear photometric or spectroscopic evidence for an accretion disc, a dominating secondary, an additional light source which could be produced by an accretion column - we consider the case of the missing HeII strong enough to assign a low probability to it.

\subsection{A dwarf nova?}

The main point which speaks against a dwarf-nova classification is the apparent absence of the disc: the lightcurve does not show any flickering, there are no recorded outbursts, the emission lines do not have the characteristic double peaks and might be produced elsewhere in the system (e.g., in an accretion column). However, in spite of showing no flickering, the lightcurve might actually contain evidence for the presence of a disc, in the fact that a) the minima are of different depth, and b) that the deep minimum occurs at the superior conjunction of the secondary. In our description of that phase (Table 5) we explained the deep minimum by an at least partly obscuration of the additional light source. At superior 
conjunction, the most probable component which could stand between the observer and the combined light of the secondary and the additional source is a (faint) accretion disc (the primary is, of course, much too small to cause the observed broad minimum).

A faint, low-mass-transfer, accretion disc could also explain both the apparent absence of flickering (because the variation is drowned in the contribution of the secondary) and of outbursts. A look into the catalogue of Ritter \& Kolb (1998) shows that the outburst recurrence times for long-period dwarf novae usually are $<100 \mathrm{~d}$, and thus rather short, but also that the recurrence intervals for most dwarf novae in this region are unknown (which might actually indicate long outburst recurrence times). There is also the case of the dwarf nova $\mathrm{CH} \mathrm{UMa}$ which, at an orbital period of $8.2 \mathrm{~h}$, has a recurrence time $\Delta t_{\text {out }} \sim 300-370 \mathrm{~d}$ (Simon 2000). The long-term monitoring of CW $1045+525$ since 1999 still consists of many gaps and has a limiting magnitude $V \sim 14$ (as shows a query in the VSNET database ${ }^{6}$ ), so that in principle short, small (up to $\Delta V \sim 1.5 \mathrm{mag}$ ) outbursts could have been occurred unnoticed. On the other hand, a reasonably dense monitoring (50 to 125 data points per year) in the years 1990-1999 at the automated 0.41-m RoboScope telescope in Indiana (e.g., Ringwald et al. 1996; and references therein) failed to show any variations larger than $0.4 \mathrm{mag}$ (Honeycutt 2001, private communication). This makes the "frequent small-amplitude outburst" scenario rather unlikely.

If the above picture applies, the single-peaked emission lines together with the photometric variation suggest a medium inclination in the range $\sim 45^{\circ}-60^{\circ}$. The additional emission source could then be interpreted as a bright spot and/or the mass stream. It appears probable that the additional continuum emission and the additional line emission do not originate from exactly the same region, and furthermore, that (at least) two additional sources contribute to the emission line profile. Evidence for this comes from the following:

- The phase difference of the extrema in the lightcurve, and especially of the minima, is not exactly 0.5 . This indicates that the additional light source is located at a certain angle from the line secondary - primary, and thus probably not very close to the centre of mass;

- The diagnostic diagram (Fig. 7) and the radial-velocity measurements of the line wings (Fig. 4) indicate an additional emission contribution also at higher velocities, as even the extreme line wings show a phase difference $\Delta \varphi \sim 0.1$ orbits with respect to the absorption lines;

- Last, but not least, there is the strong emission component near the centre of mass, which can therefore not be very much displaced from the line which connects the primary and the secondary.

\footnotetext{
${ }^{6}$ Available on the $\mathrm{WWW}$ at

http://www.kusastro.kyoto-u.ac.jp/vsnet/etc/searchobs . cgi?text=CW1045\%2B525
}

While the additional continuum source and the "highvelocity" emission might well stem from the same location, which could be explained as a "classic" bright spot (a phase difference of 0.1 translates to an angle of $36^{\circ}$ ), the origin of the "stationary" component remains unknown. Time-resolved high-resolution spectroscopy would thus be welcome for a detailed examination of the line profile and its individual components.

\section{Summary}

1. We have presented photometric and spectroscopic data of the cataclysmic variable CW $1045+525$.

2. The spectrum shows emission lines of the Balmer and the He I series, as well as the absorption spectrum of the late-type secondary.

3. Comparison with template spectra yielded best agreements for a secondary spectral type of K5V-M0V contributing $\sim 70 \%$ of the light in the optical range.

4. The orbital period was derived by radial-velocity measurements of the absorption lines of the secondary. The covered range of $495 \mathrm{~d}$ constrains the period to $P_{\text {orb }}=$ $0.271278(1) \mathrm{d}$, without any cycle-count ambiguity.

5. The emission lines have an asymmetric profile whose variations clearly show an orbital modulation. It appears to be composed out of at least two components: an almost stationary line centre and "highvelocity" wings which move with a semi-amplitude of $\sim 130 \mathrm{~km} \mathrm{~s}^{-1}$. The latter show a phase displacement of $\sim 0.1$ orbits with respect to the absorption lines.

6. The photometric lightcurve is dominated by the ellipsoidal variation of the secondary, but also includes light from an additional "asymmetric" source. The precision of the derived period allowed an extrapolation to the photometric data and thus an orbital phasing of the features in the lightcurve.

7. Neither the spectroscopy nor the photometry shows clear evidence for the presence of an accretion disc, although certain lightcurve features can be interpreted as the obscuration of the additional light source and the secondary by a faint disc.

8. The secondary star's brightness and spectral type yield a distance range $350-700$ pc. The velocity amplitude of the absorption spectrum does not suggest anything extraordinary about the component masses.

9. We tentatively conclude that CW $1045+525$ is a longperiod dwarf nova with a faint accretion disc and a long outburst recurrence time.

Acknowledgements. J.R.T. and W.H.F. thank the U. S. National Science Foundation for support through grant AST 9987334, and the MDM Observatory staff for their help. C.T., N.B., and L.S. thank the director of the Hoher List Observatory, W. Seggewiss, for generous allocation of observing time, and the Asiago staff for their support. We also thank R.K. Honeycutt for his helpful comments and the information on the RoboScope monitoring. 


\section{References}

Baraffe, I., \& Kolb, U. 2000, MNRAS, 318, 354

Bennert, N., Kleimann, J., König, I., et al. 1999, Astron. Ges. Abstr. Ser., 15, P46

Beuermann, K., Baraffe, I., Kolb, U., \& Weichhold, M. 1998, A\&A, 339, 518

Beuermann, K., Baraffe, I., \& Hauschildt, P. 1999, A\&A, 348, 524

Burwitz, V., Reinsch, K., Schwope, A. D., et al. 1998, A\&A, 331,262

Downes, R. A., \& Shara, M. M. 1993, PASP, 105, 127

Henden, A. A., \& Honeycutt, R. K. 1995, PASP, 107, 324

Horne, K., \& Marsh, T. R. 1986, MNRAS, 218, 761

Kurtz, M. J., \& Mink, D. J. 1998, PASP, 110, 934

Martínez-Pais, I. G., Martín-Hernánde, Casares, J., \& Rodríguez-Gil, P. 2000, ApJ, 538, 315

Mennickent, R. E., \& Tappert, C. 2001, A\&A, 372, 563

Pesch, P., \& Sanduleak, N. 1987, IBVS, 2989

Pickles, A. J. 1998, PASP, 110, 863

Ringwald, F. A., Thorstensen, J. R., Honeycutt, R. K., \& Smith, R. C. 1996, AJ, 111, 2077

Ritter, H., \& Kolb, U. 1998, A\&AS, 129, 83

Scargle, J. D. 1982, ApJ, 263, 835
Schmidtobreick, L., Bennert, N., König, I., et al. 2000, in Proceedings of the Ph.D. Conference on Variable Stars (Kecskemét, Hungary), in press

Schneider, D. P., \& Young, P. 1980, ApJ, 238, 946

Schwarzenberg-Czerny, A. 1989, MNRAS, 241, 153

Shafter, A. W. 1983, ApJ, 267, 222

Simon, V. 2000, A\&A, 354, 103

Stover, R. J. 1981, ApJ, 248, 684

Szkody, P., \& Howell, S. B. 1992, ApJS, 78, 537

Tappert, C. 1999, Ph.D. Thesis, Ruhr-Universität Bochum

Tappert, C., Bennert, N., Schmidtobreick, L., \& Bianchini, A. 2000, Astron. Ges. Abstr. Ser., 17, P25

Taylor, C. J., Thorstensen, J. R., \& Patterson, J. 1999, PASP, 111,184

Thorstensen, J. R., \& Freed, I. W. 1985, AJ, 90, 2082

Thorstensen, J. R., Patterson, J. O., Shambrook, A., \& Thomas, G. 1996, PASP, 108, 73

Tonry, J., \& Davis, M. 1979, AJ, 84, 1511

Wade, R. A., \& Horne, K. 1988, ApJ, 324, 411

Wagner, R. M., Sion, E. M., Liebert, J., \& Starrfield, S. G. 1988, ApJ, 328, 213

Warner, B. 1995, Cataclysmic Variable Stars (Cambridge University Press) 